\title{
Neuro-immune crosstalk and allergic inflammation
}

\author{
Hiroki Kabata and David Artis \\ jill Roberts Institute for Research in Inflammatory Bowel Disease, Joan and Sanford I. Weill Department of Medicine, Department of Microbiology and Immunology, Weill Cornell Medicine, Cornell University, \\ New York, New York, USA

\begin{abstract}
The neuronal and immune systems exhibit bidirectional interactions that play a critical role in tissue homeostasis, infection, and inflammation. Neuron-derived neuropeptides and neurotransmitters regulate immune cell functions, whereas inflammatory mediators produced by immune cells enhance neuronal activation. In recent years, accumulating evidence suggests that peripheral neurons and immune cells are colocalized and affect each other in local tissues. A variety of cytokines, inflammatory mediators, neuropeptides, and neurotransmitters appear to facilitate this crosstalk and positivefeedback loops between multiple types of immune cells and the central, peripheral, sympathetic, parasympathetic, and enteric nervous systems. In this Review, we discuss these recent findings regarding neuro-immune crosstalk that are uncovering molecular mechanisms that regulate inflammation. Finally, neuro-immune crosstalk has a key role in the pathophysiology of allergic diseases, and we present evidence indicating that neuro-immune interactions regulate asthma pathophysiology through both direct and indirect mechanisms.
\end{abstract}

\section{Introduction}

Epithelial barrier cells cover the surface of the entire body. These cells are constantly monitored by tightly regulated immune and neuronal cell networks. Environmental pathogens and allergens trigger the rapid release of proinflammatory cytokines from the epithelial barrier, which leads to the induction of innate immune responses followed by adaptive immune responses. At the same time, neuronal systems sense tissue damage or stimulation and transmit the information to the central nervous system (CNS) or nearby efferent neurons in visceral organs, resulting in various neuronal symptoms, such as itch, sneezing, cough, bronchoconstriction, dysphasia, and altered gastrointestinal motility. These collaborative immune and neuronal responses can contribute to the efficient elimination of infection and noxious stimuli and maintain tissue homeostasis.

Importantly, these immune and neuronal systems are not operating separately. Rather, they closely interact with each other. Activated immune cells produce inflammatory mediators, such as cytokines, lipid mediators, and histamine, which directly stimulate the terminals of peripheral nerves in local tissues. These stimulations not only transmit to the CNS but also enhance neuronal excitability and lower the activation threshold of neurons in response to stimuli, allowing subthreshold or even non-noxious stimuli to evoke neuronal activation and enhance neuronal function in sites of inflammation. In addition, the nerve terminals release various neuropeptides upon neuronal activation, and the released neuropeptides have a significant regulatory effect on multiple immune cells. Early experiments using electrical vagus nerve stimulation demonstrated that vagus nerve-derived acetylcholine suppressed the release of inflammatory cytokines from macrophages and dampened the endotoxin-induced systemic inflammatory response (1). Similarly,

Conflict of interest: The authors have declared that no conflict of interest exists. Copyright: () 2019 American Society for Clinical Investigation

Reference information: J Clin Invest. 2019;129(4):1475-1482.

https://doi.org/10.1172/JCl124609. sensory nerve terminals contain various neuropeptides, such as substance $\mathrm{P}$, neurokinin A, vasoactive intestinal peptide (VIP), and calcitonin gene-related peptide (CGRP), which directly regulate immune cell functions (2). In addition, these neuropeptides also affect endothelial cells and smooth muscle cells, leading to edema, vasodilation, and smooth muscle contraction and relaxation. These changes can further enhance immune cell responses, albeit indirectly. Thus, immune and neuronal systems closely interact with each other with profound effects on immunity, inflammation, and tissue homeostasis.

In recent years, research regarding neuro-immune crosstalk has focused increasingly on the neuro-immune interactions acting locally in tissues. Accumulating evidence has revealed the close anatomical contact and interaction between peripheral neurons and immune cells in peripheral tissues (3, 4). Apart from their systemic effects, various immune cells, including dendritic cells (DCs), macrophages, mast cells, and innate lymphoid cells (ILCs), share anatomical localization with peripheral neurons, and local neuro-immune interactions may have a potent effect on tissue homeostasis and inflammation. Specifically, group 2 innate lymphoid cells (ILC2s) are a recently identified lymphoid cell population that produces type 2 cytokines, including IL-5 and IL-13, in response to epithelial cell-derived cytokines, such as IL-33 and IL-25 (5-7). Neuronal regulation of ILC2s may play an important role in the induction of type 2 inflammation in the context of allergic diseases.

In this article, we first review the general concepts underlying neuro-immune interaction, then introduce recent evidence regarding the influence of local neuro-immune interactions. Lastly, we discuss the influence of neuro-immune interactions in the pathophysiology of asthma.

\section{General neuro-immune interactions}

The central nervous system (CNS) and peripheral tissues are connected by the peripheral nervous system (PNS), which transmits 


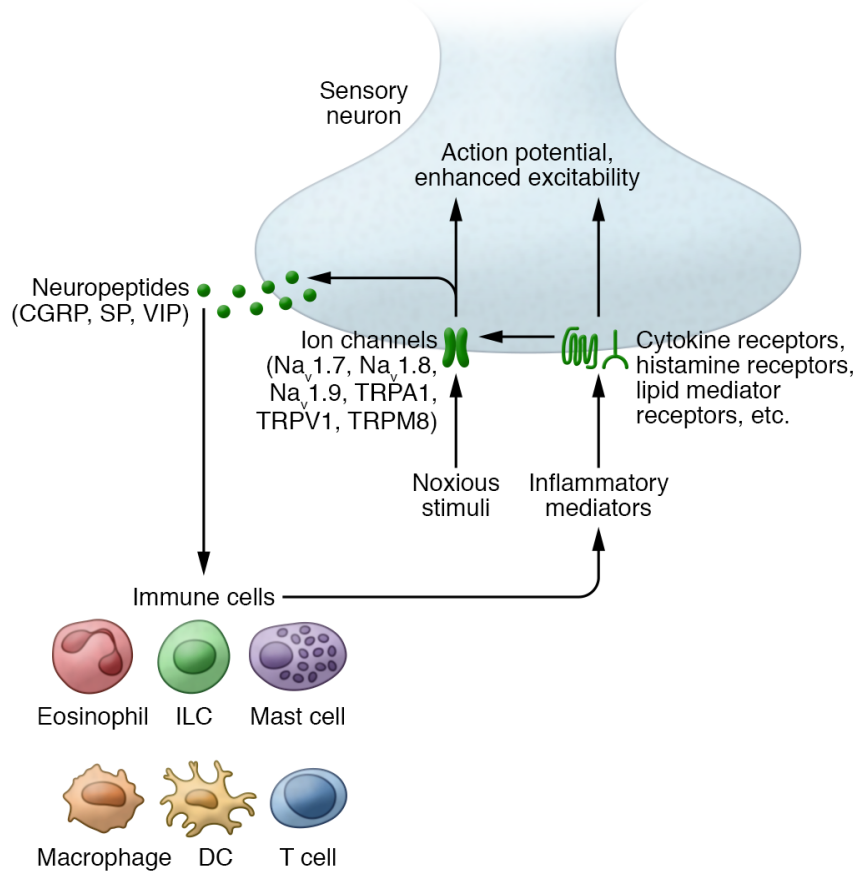

Figure 1. The interaction between sensory neurons and immune cells. Immune cell-derived inflammatory mediators and noxious stimuli activate neurons via ion channels and various receptors for these mediators. Activated neurons release neuropeptides, which directly affect immune cells and regulate inflammatory responses. SP, substance $P$.

a variety of signals via neurotransmitters and neuropeptides. The PNS is divided into somatic, autonomic, and enteric nervous systems. The autonomic nervous system is further divided into the sympathetic nerves and the parasympathetic nerves. The PNS has various regulatory effects on immune reactions via neurotransmitters and neuropeptides.

The peripheral sympathetic nerves release noradrenaline (also called norepinephrine), and adrenal glands produce adrenaline (also called epinephrine). Multiple immune cells express the receptors for noradrenaline and adrenaline, $\alpha$-adrenoceptors and $\beta$-adrenoceptors, and are directly stimulated by these catecholamines. In general, sympathetic nerves promote inflammation in the initial phase of immune reactions, whereas they suppress inflammation in the later phase (8). Specifically, adrenaline and noradrenaline mainly suppress the function of immune cells, such as neutrophils, natural killer (NK) cells, and monocytes, via $\beta$-adrenoceptors. Additionally, these neurotransmitters induce DCs to decrease Th1 cell differentiation and promote Th2 cell differentiation (9). However, these responses are variable depending on the local environment, including catecholamine concentrations, receptor expression levels, and costimulation (8).

The parasympathetic nerves, on the other hand, produce acetylcholine, which directly affects immune cells via muscarinic and nicotinic acetylcholine receptors. Acetylcholine has an antiinflammatory effect and suppresses the activation of macrophages, basophils, and mast cells via $\alpha 7$-nicotinic acetylcholine receptors ( $\alpha$ 7nAChRs) (10). However, acetylcholine also stimulates muscarinic acetylcholine receptors and is capable of promoting Th2 cell-polarizing effects via DCs and $C D 4^{+} \mathrm{T}$ cells (10). Therefore, the effect of parasympathetic nerves, like that of sympathetic nerves, is modified depending on the local environment and target cell receptor expression.

Sensory neurons are heterogeneous with respect to their sensitivity to stimuli, conduction velocity (myelination), and neuropeptide content. Each sensory nerve terminal expresses various combinations of ion channels to sense a variety of stimulations, including $\mathrm{Na}_{v} 1.7, \mathrm{Na}_{v} 1.8, \mathrm{Na}_{v} 1.9$, transient receptor potential vanilloid 1 (TRPV1), transient receptor potential ankyrin 1 (TRPA1), and transient receptor potential cation channel subfamily M member 8 (TRPM8) (Figure 1). TRPV1 is responsive to high temperature and capsaicin (11), whereas TRPA1 mainly responds to chemical and mechanical stress as well as chemical irritants, including wasabi, and cold temperature $(12,13)$. TRPM8 is responsive to cold temperature and menthol (14). A specialized subset of sensory neurons detecting noxious or potentially harmful stimuli are called nociceptors, which innervate skin, joints, respiratory, and gastrointestinal tract (15). Most nociceptors are small-diameter, unmyelinated, slowly conducting nerves referred to as C-fibers. Nociceptors express not only TRPA1 and TRPV1 but also various receptors for cytokines, lipid mediators, and growth factors, including ATP, adenosine, 5-hydroxytryptamine, cysteinyl leukotrienes, and protease-activated receptors. Therefore, a variety of stimulants, including inflammatory mediators, leads to the activation of nociceptors through these receptors (Figure 1). For example, type 2 cytokines, such as IL-4, IL-5, and IL-13, induce sensory nerve activation and induce chronic itch (16). In addition, thymic stromal lymphopoietin (TSLP) has recently been found to activate TRPA1 by binding to its receptor, TSLPR, on sensory nerves in the skin of atopic dermatitis patients (17). Furthermore, Th2 cell-derived IL-31 activates TRPV1 ${ }^{+}{ }^{T R P A} 1^{+}$ sensory nerves and induces mast cell-independent itch (18).

Notably, the terminals of nociceptors contain neuropeptides, such as CGRP, substance P, and VIP, which are rapidly released in response to noxious stimuli and inflammation. These neuropeptides directly act on various immune cells (Figure 1). Substance $\mathrm{P}$ is known to be a proinflammatory neuropeptide that activates multiple immune cells, including T cells, macrophages, DCs, mast cells, eosinophils, and neutrophils (19-21). The functions of VIP and CGRP skew toward a Th2 cytokine-like phenotype. Moreover, VIP suppresses inflammatory cytokines derived from DCs and macrophages, whereas it promotes Th2 cell differentiation, survival, and migration (22-24), and CGRP induces mast cell degranulation and shifts Langerhans cells to promote Th2 differentiation (25). These neuropeptides also affect nonimmune cells and increase vascular permeability, which is involved in the further recruitment of immune cells.

Thus, neuropeptides and neurotransmitters have a wide range of regulatory effects on multiple immune cells, and immune cellderived mediators induce neuronal activation, which leads to a further release of neuropeptides in a positive-feedback loop. In addition, many observations suggest that these effects are modified by the local environment. Therefore, there has been a recent increased interest in studying the local effect of neuro-immune communications.

\section{Local neuro-immune interaction in peripheral tissues}

Although neuropeptides and neurotransmitters have multiple regulatory functions in the immune system, recent studies have 

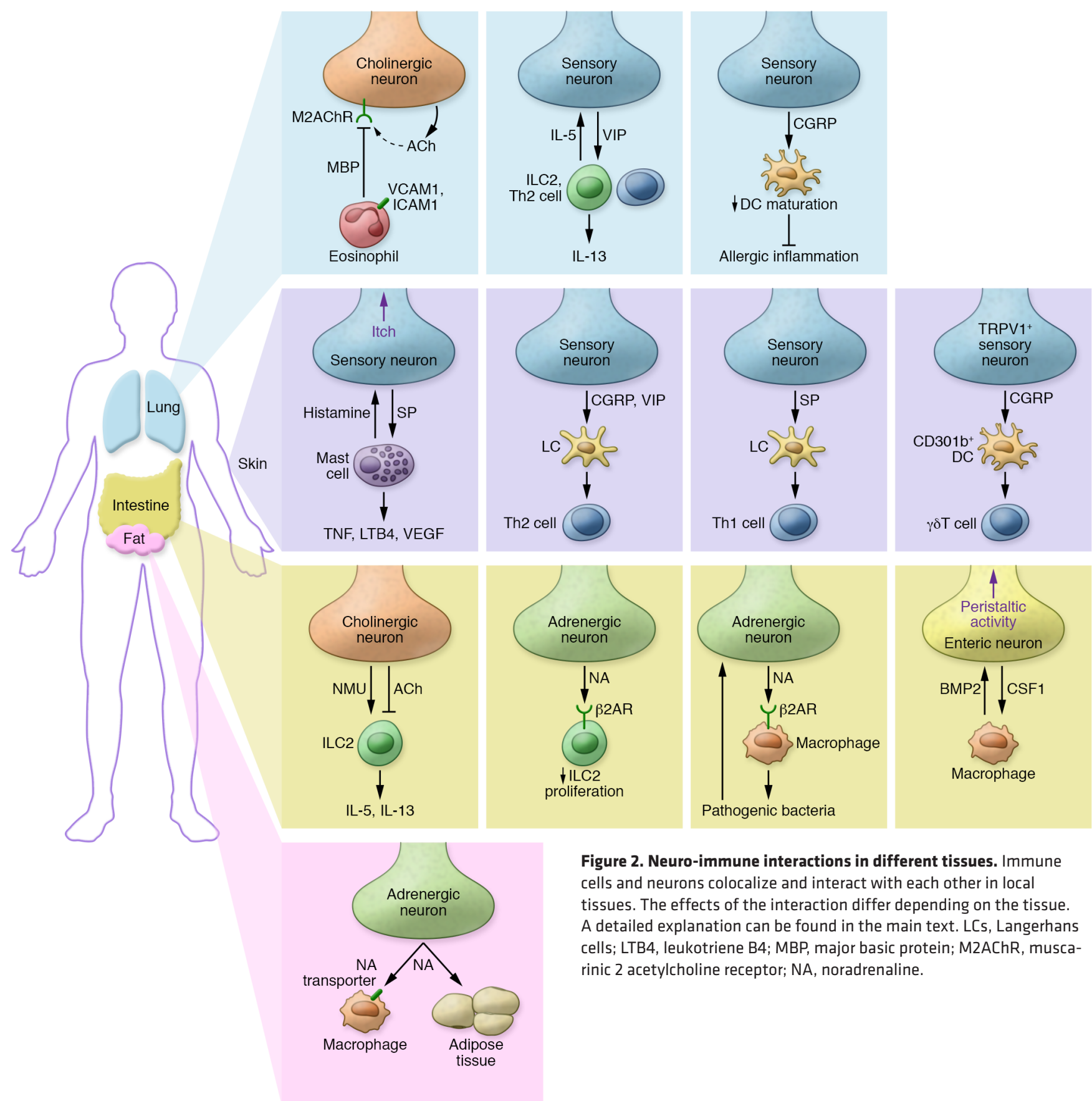

Figure 2. Neuro-immune interactions in different tissues. Immune cells and neurons colocalize and interact with each other in local tissues. The effects of the interaction differ depending on the tissue. A detailed explanation can be found in the main text. LCs, Langerhans cells; LTB4, leukotriene B4; MBP, major basic protein; M2AChR, muscarinic 2 acetylcholine receptor; NA, noradrenaline.

emphasized the importance of the anatomical relationship between neurons and immune cells in the peripheral tissues, including skin, lungs, and intestine, and accumulating evidence suggests that various immune cells and neurons directly interact with each other in these sites (refs. 3, 4, and Figure 2).

Neuronal regulation of granulocyte responses. Many reports have shown that mast cells closely localize with sensory nerves in the gastrointestinal tract, urinary bladder, and skin (26-28). This anatomic colocalization between mast cells and nerves becomes more prevalent in the context of inflammation such as atopic dermatitis (29). The epidermis and papillary dermis of an atopic dermatitis lesion is hyperinnervated with increased sub- stance P-positive nerve fibers, with increased mast cells expressing substance P's receptor (NK-1R) compared with nonlesional skin. This finding implies that innervation and neuropeptides play a role in the pathogenesis of AD (30-32). Sensory nervederived substance $P$ induces mast cell degranulation and release of histamine, TNF- $\alpha$, leukotriene $\mathrm{B}_{4}$, and vascular endothelial growth factor (VEGF) (ref. 33 and Figure 2). VEGF further promotes endothelial cell proliferation and vascularization, facilitating the inflammatory process. Notably, mast cell-derived histamine acts on the histamine receptors on sensory nerve endings and evokes further release of neuropeptides, which establishes a positive-feedback loop between mast cells and sensory nerves in 
tissues (ref. 34 and Figure 2). Mast cells also release neurotrophic factors that promote local nerve elongation (35). Furthermore, substance P-mast cell pathways induce itch, and the administration of an NK-1R antagonist decreases scratching behavior in mice (36). Collectively, these findings suggest that the close interaction between mast cells and neurons in tissues affects inflammatory responses.

In addition to mast cells, other granulocytes are less clearly colocalized with neurons at a steady state. However, eosinophils localize with airway cholinergic nerves after allergen challenges. It has been suggested that eosinophils adhere to cholinergic nerves via vascular cell adhesion molecule 1 (VCAM1) and intercellular adhesion molecule 1 (ICAM1) (ref. 37 and Figure 2). The terminals of cholinergic nerves express the M2 muscarinic acetylcholine receptor, and stimulation of the receptor limits further release of acetylcholine in a negative-feedback pathway. Eosinophil-derived major basic protein acts as an antagonist of M2 muscarinic receptors. Therefore, eosinophil-derived major basic protein inhibits the negative-feedback loop and potentiates overproduction of acetylcholine, which enhances acetylcholine-induced bronchoconstriction in the airway (ref. 38 and Figure 2). In addition, it is also reported that sensory nerve-derived neuropeptides such as substance P, CGRP, secretoneurin, VIP, and secretin promote the migration of eosinophils (39), which play a role in increasing sensory nerve responsiveness, stimulating nerve growth, and increasing neuropeptide expression in the context of inflammation (40). Therefore, the interactions between eosinophils and neurons may accelerate inflammation.

Neuro-immune interactions with myeloid cells. Recent studies have revealed a previouslyunrecognized local neuro-immuneinteraction between macrophages and neurons in the intestine and adipose tissues. Enteric neurons produce colony-stimulating factor 1 (CSF1), a growth factor required for the development of MHC class $\mathrm{II}^{+} \mathrm{CX} 3 \mathrm{CR} 1^{+}$macrophages in the intestinal muscularis externa. These muscularis macrophages regulate peristaltic activity, a muscular contraction that moves food down the intestine, through the production of bone morphogenetic protein 2 (BMP2). Macrophage-derived BMP2 stimulates BMP receptor (BMPR) on enteric neurons to induce CSF1 secretion, which leads to the further growth of macrophages in a positive-feedback loop. Therefore, these interactions between enteric neurons and macrophages play an important role in the homeostasis of the intestine (ref. 41 and Figure 2). Data indicate that this neuro-macrophage crosstalk is further enhanced by the microbiome. In contrast, intestinal pathogenic bacterial infections stimulate enteric sympathetic neurons to produce noradrenaline. The noradrenaline directly stimulates muscularis macrophages via $\beta_{2}$-adrenergic receptors ( $\beta 2 \mathrm{ARs}$ ) and promotes a tissue-protective function (ref. 42 and Figure 2). Furthermore, a recent study demonstrated that macrophages mediate noradrenaline clearance via the noradrenaline transporter and production of noradrenaline degradation enzymes in adipose tissue. The sympathetic nervous system also plays an essential role in the regulation of metabolic homeostasis (43-45). Loss of function of the noradrenaline transporter on macrophages increases noradrenaline's effects, which promotes increases in brown adipose tissue (BAT) content. Elevated BAT levels enhance thermogenesis and lead to weight loss in obese mice (46). Another study demon- strated that BAT-resident macrophages are related to the maintenance of sympathetic innervation in BAT (44). Thus, the interaction between sympathetic neurons and macrophages is involved in metabolic regulation in adipose tissues (Figure 2). Taken together, the interaction between neurons and macrophages has multiple effects depending on the tissue microenvironment.

DCs are also affected by neuropeptides and are in close contact with sensory neurons in the airway and skin $(47,48)$. Sensory nerve-derived CGRP inhibits DC maturation, which leads to suppression of $\mathrm{T}$ cell activation. Another study showed that adoptive transfer of CGRP-treated DCs diminishes allergic airway inflammation in vivo, which was associated with reduced eosinophilia and elevated levels of IL-10 in bronchoalveolar lavage fluid (49). These findings suggest that sensory neuron-derived CGRP has a suppressive effect on DCs in the lung (Figure 2). Again, however, the neuronal regulatory effects are variable depending on the tissue. In the skin, DCs are in close contact with TRPV1 ${ }^{+}$sensory neurons (48). CGRP derived from TRPV1+ neurons drives IL-23 production from CD301 $\mathrm{b}^{+} \mathrm{DCs}$, leading to secretion of $\gamma \delta \mathrm{T}$ cell-derived IL-17A, which inhibits cutaneous Candida albicans infection (ref. 50 and Figure 2). Furthermore, Langerhans cells, a population of DCs in the epidermis of the skin, are also very closely associated with epidermal sensory nerves (51). CGRP and VIP enhance Langerhans cell antigen presentation on Th2 cells while inhibiting presentation to Th1 cells (refs. 51, 52, and Figure 2). In contrast, substance $P$ enhances Langerhans cell migration and antigen presentation, leading to the generation of Th1-type immune response (ref. 53 and Figure 2). Together, these studies indicate that DCs are closely interacting with sensory neurons in the lungs and skin, and their regulatory effects depend on the tissue microenvironment.

Neuro-immune interactions with ILCs. ILCs are a recently discovered lymphoid cell population that lack rearranged antigen receptors and produce a variety of cytokines. ILCs are classified into three groups on the basis of transcriptional factors and production of cytokines: ILC1s (expressing T-bet, producing IFN- $\gamma$ ), ILC2s (expressing GATA3, producing IL-5 and IL-13), and ILC3s (expressing ROR $\gamma$ t, producing IL-17 and/or IL-22) (54).

Recent studies shed light on the new mechanisms of neuronal regulation of ILC2s. These cells produce type 2 cytokines in response to epithelial cell-derived cytokines, including TSLP, IL-33, and IL-25, and play a critical role in eosinophilic inflammation of allergic diseases (55). A series of seminal studies have demonstrated that tuft cells, chemosensory cells that express TRPM5 to sense bitter and sweet taste, are the dominant sources of IL-25 in the respiratory tract and intestine (56-59). ILC2s are predominantly tissue-resident cells and reside in various tissues at a steady state. Recent studies have revealed that ILC2s are closely associated with neurons in the intestine and that neuropeptides and neurotransmitters directly activate or suppress ILC2 function. For example, ILC2s colocalize with neuromedin Uexpressing (NMU-expressing) neurons, most of which are enteric cholinergic neurons in the intestine (Figure 2). ILC2s selectively express NMU receptor 1 (NMUR1), and NMU has a potent effect of inducing the proliferation of ILC2s as well as inducing their production of the cytokines IL-5, IL-9, IL-13, and AREG (60-62). NMU enhances type 2 inflammation via the activation of ILC2s 
in helminth infection and house dust mite-induced (HDMinduced) asthma model mice $(60,62)$. Enteric cholinergic neurons also produce acetylcholine, and a recent study revealed that ILC2s express $\alpha 7 \mathrm{nAChR}$ (63). $\alpha 7 \mathrm{nAChR}$ stimulation suppresses GATA3 and NF- $\mathrm{KB}$ signaling pathways in ILC2s, which decreases their production of type 2 cytokines (63). Thus, enteric cholinergic neurons are capable of both activating and suppressing the function of ILC2s depending on the specific neuropeptide or neurotransmitter. In addition, it was reported that IL-33 and the excretory/ secretory products of the gastrointestinal roundworm Nippostrongylus brasiliensis directly induce NMU production from cholinergic neurons in a MyD88-dependent manner (62). Therefore, the role of enteric cholinergic neurons in the regulation of ILC2s is altered depending on exposure to specific pathogens and allergens.

Furthermore, a recent study demonstrated that ILC2s also contact enteric adrenergic neurons in the intestine. $\beta 2 \mathrm{AR}$ signaling suppresses ILC2 proliferation cell-intrinsically and suppresses ILC2-mediated inflammation. Consistent with this, $\beta 2 A R-$ deficient mice exhibit more severe ILC2-mediated type 2 inflammation in the intestine and lungs (ref. 64 and Figure 2). These data suggest that physiological catecholamine suppresses ILC2s and regulates type 2 inflammation. In addition, a recent study demonstrated that pulmonary neuroendocrine cells (PNECs) are important sources of CGRP (65). PNECs are rare airway epithelial cells that sense various stimulations, including oxygen, stretch, and chemicals, as well as release neuropeptides. PNECs and ILC2s are colocalized near airway branch points in the lungs. ILC2s express CGRP receptors, and CGRP induces IL- 5 and IL- 6 production from ILC2s in the presence of IL-7 + IL-33 or IL-7 + IL-25 + IL-33 (65). Furthermore, ILC2s also express VIP receptor, and VIP has activating effects on ILC2s. For example, VIP induces IL-5 production from ILC2s in the presence of IL-7 (66). Collectively, ILC2s are directly affected by neuropeptides and neurotransmitters in the local tissues, including the lungs and the intestine.

ILC3s are also adjacent to enteric glial cells, the supportive cells for enteric neurons, in the intestine. Bacterial infection and tissue damage activate these glial cells to secrete glial-derived neurotrophic factor family ligands (GFLs) via TLR signals and alarmins, including IL-1 $\beta$ and IL-33, in an MyD88-dependent manner. GFLs directly induce ILC3s to release the tissue-protective cytokine IL-22 via the tyrosine kinase receptor RET. GFL-induced RET activation leads to rapid ERK1/2, AKT, p38 MAP kinase, and STAT3 phosphorylation as well as increased $I l 22$ transcription in ILC3s (67). Thus, the tissue-protective role of ILC3 is regulated by enteric glial cells in the intestine.

Together, these findings highlight that neuro-immune interactions in the local tissue are very complicated and varied depending on the tissues, cell types, and inflammatory or infectious conditions. Therefore, the general roles of neuro-immune interactions are not always applicable to local neuro-immune interactions, and it is necessary to consider the influence of these local interactions in the context of specific diseases.

\section{Neuronal regulation in asthma}

Classically, neuronal systems, especially sympathetic and parasympathetic nerves, were thought to affect smooth muscle cells and contribute to the symptoms of asthma. However, as evidence of neuro-immune interactions in tissues accumulates, it has been suggested that neuronal systems directly affect immune responses in asthma. The immune responses in asthma mainly divide into two distinct cascades: allergen-specific and allergen-nonspecific responses. In allergen-specific responses, allergens are captured by antigen-presenting cells like DCs that induce antigenspecific Th2 cells in the lymph nodes. Then, allergens activate antigen-specific Th2 cells, which leads to the production of type 2 cytokines such as IL-4, IL-5, and IL- 13 . IL- 4 stimulates B cells to synthesize IgE antibodies, IL-5 induces eosinophilic inflammation, and IL-13 promotes mucin overproduction and tissue remodeling. Moreover, IgE causes antigen-specific activation of mast cells and basophils, which results in the release of inflammatory mediators. On the other hand, some allergens directly induce the release of cytokines such as IL-33 and IL-25 from epithelial cells, and these cytokines directly activate ILC2s independent of antigen-specificity. Activated ILC2s produce type 2 cytokines like IL-5 and IL-13 and cause eosinophilic inflammation without acquired immunity. Subsequently, ILC2s promote the induction of antigen-specific Th2 cells. Although neuronal systems modify both allergen-specific and allergen-nonspecific immune responses in multidimensional ways, recent studies demonstrated that they might have more impact on ILC2-mediated allergen-nonspecific immune responses.

Neuronal regulation in asthma by sympathetic nerves. Sympathetic nerve-derived noradrenaline is known to be involved in bronchial muscle relaxation via $\beta 2 \mathrm{AR}$ on airway smooth muscle cells, and agonists of $\beta 2 \mathrm{AR}$ are used for the treatment of asthma and chronic obstructive pulmonary disease (COPD) as a bronchodilator. However, some studies report that this neuronal regulation is modified by immune cells. Mast cells infiltrate in airway smooth muscles of patients with asthma (68), and mast cell-derived TGF- $\beta$ induces phosphorylation of $\beta 2$ AR on smooth muscle cells, which results in a reduced response to a $\beta 2 A R$ agonist (69). In addition, noradrenaline can directly suppress proinflammatory cytokine production from immune cells through $\beta 2 A R$, and $\beta 2$ AR-deficient mice have more severe type 2 airway inflammation in allergen-nonspecific asthma mouse models. Specifically, $\beta 2 A R$ agonists dampen IL-33- and Alternaria-induced airway inflammation via suppression of ILC2s (64). Thus, $\beta 2 A R$ agonists might have a beneficial effect on asthma via not only smooth muscle cells but also immune cells (Figure 3). However, it is still unclear whether $\beta 2 \mathrm{AR}$ agonists can suppress human ILC2s and suppress ILC2-mediated inflammation in patients with asthma, although it is reported that human lung ILC2s express $\beta 2 A R(64)$.

Neuronal regulation in asthma by parasympathetic nerves. Parasympathetic nerve-derived acetylcholine induces airway smooth muscle contraction and mucus secretion mainly via muscarinic M3 receptors. Therefore, inhalation of selective M3 receptor antagonists, which act as bronchodilators, is currently used for the treatment of asthma and COPD (70). Patients with asthma have increased pulmonary cholinergic nerve activity and hypersensitivity to cholinergic agonists. As described earlier, eosinophil-derived major basic protein mediates the dysfunction of the muscarinic M2 receptor, which might contribute to this increased cholinergic neuron activity (38). On the immune side, vagal nerve stimulation suppresses the inflammatory response 
A

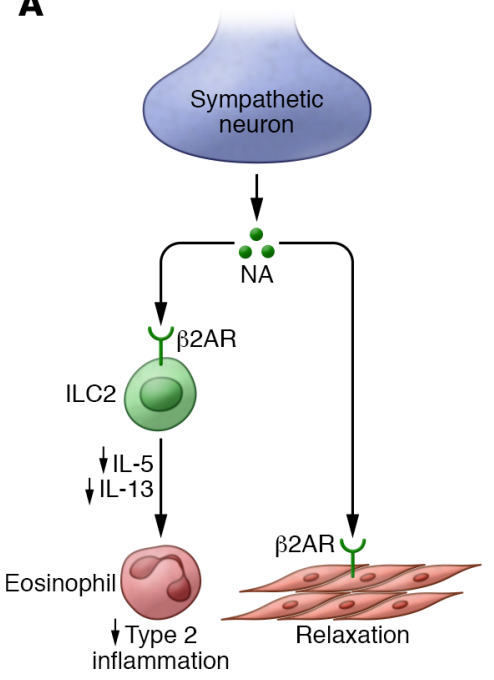

B

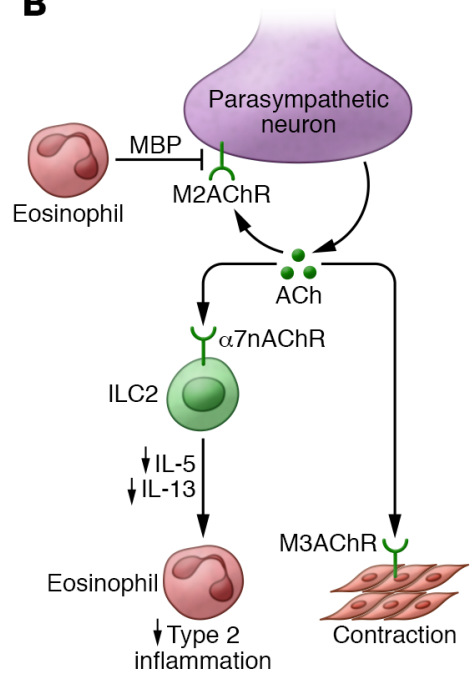

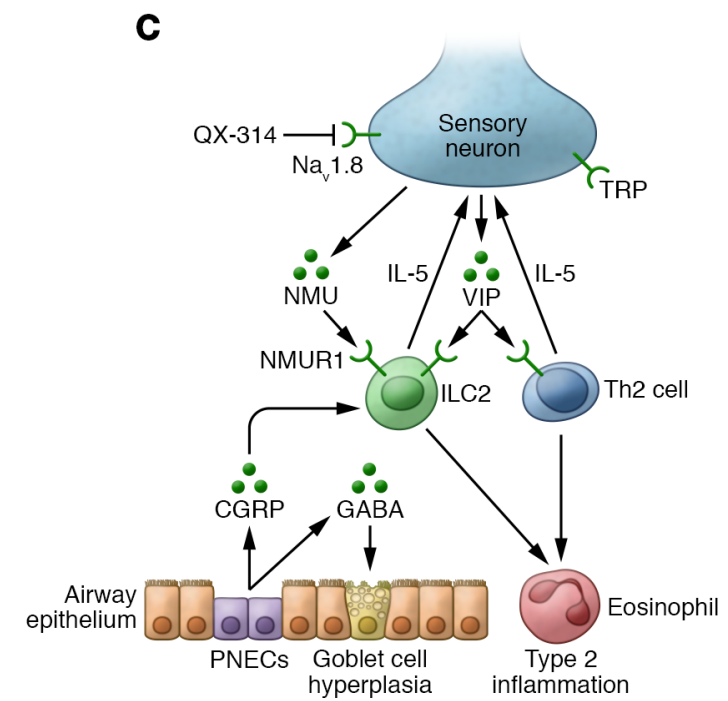

Figure 3. The neuronal regulation of asthma. Sympathetic neuron-derived NA suppresses ILC2-mediated type 2 inflammation via $\beta 2 A R s$ located on ILC2s and induces the relaxation of smooth muscle cells. Parasympathetic neuron-derived acetylcholine (ACh) suppresses ILC2s via $\alpha 7 A C h R$ and induces the contraction of smooth muscle cells via muscarinic 3 acetylcholine receptor (M3AChR). Eosinophils block the M2AChR suppressing the negative feedback loop of ACh. Sensory neuron-derived neuropeptides, including VIP, activate ILC2s and Th2 cells, which enhances type 2 inflammation. IL-5 activates the terminals of sensory neurons and induces the further release of VIP. NMU also directly activates ILC2s, leading to type 2 inflammation. Blocking the activation of sensory neurons using QX-314, a voltage-gated sodium channel blocker, inhibits the release of neuropeptides and attenuates type 2 inflammation. PNECs also produce CGRP and CABA, thereby activating ILC2s and directly inducing goblet cell hyperplasia in the airway. TRPs, transient receptor potential channels.

(1), while vagal denervation worsens lung infection, inflammation, and injury with increased proinflammatory cytokines (71). These effects are caused by acetylcholine via $\alpha 7 \mathrm{nAChR}$, and a recent study showed that $\alpha 7 n A C h R$ agonists directly suppressed ILC2s, resulting in decreased type 2 inflammation in IL-33- and Alternaria-induced airway inflammation (63). Therefore, acetylcholine has a beneficial effect on type 2 inflammation in asthma via $\alpha 7 \mathrm{nAChR}$, although it causes bronchoconstriction via muscarinic M3 receptors. Thus, parasympathetic neurons have multiple functions in the pathophysiology of asthma that vary depending on their receptors (Figure 3). Further studies are needed to clarify the clinical relevance of the interaction between cholinergic neurons and the immune system in asthma.

Sensory nerves and neuroendocrine cells regulate asthma pathophysiology. Recent studies have shown that sensory nerves also play a critical role in the pathophysiology of asthma $(72,73)$. Sensory neurons contain various neuropeptides, including substance P, VIP, and CGRP, and allergen provocation induces neuropeptide release. Indeed, it is reported that the levels of substance $\mathrm{P}$ in induced sputum are increased and correlated with airway obstruction in asthmatic patients (74). Furthermore, CGRP is elevated in the bronchoalveolar lavage fluid of asthmatics during the latephase reactions provoked by allergen inhalation (75). In recent years, several studies in mice demonstrated that blocking sensory nerves ameliorates type 2 inflammation in the airways. For example, genetic ablation of TRP-expressing sensory nerves by diphtheria toxin suppresses airway hyperreactivity in an OVA-induced allergen-specific asthma mouse model (72). In addition, genetic ablation of $\mathrm{Na}_{\mathrm{v}} 1.8^{+}$sensory nerves or QX-314 treatment (which both target a voltage-gated sodium channel to induce nociceptor silencing) reduces immune cell infiltration and airway hyperreac- tivity in an OVA-induced asthma mouse model (73). These studies suggest that allergen provocation induces the release of neuropeptides from sensory nerves and these neuropeptides directly affect immune cells. Specifically, ILC2- and $\mathrm{CD}_{4}{ }^{+} \mathrm{T}$ cell-derived IL-5 directly stimulates pulmonary nociceptors to release VIP, and VIP activates ILC2s and CD $4^{+} T$ cells to further enhance type 2 inflammation in OVA- and HDM-induced asthma mouse models in a positive-feedback loop (ref. 73 and Figure 3).

In addition, sensory nerve endings express a variety of ion channels. Capsaicin, a selective TRPV1 agonist, activates lung nociceptors to enhance OVA-induced lung inflammation via the release of neuropeptides (73). However, TRPV1 deficiency does not affect the phenotype of asthma mouse models $(72,76)$. On the other hand, TRPA1-deficient mice or treatment with HC-030031, a selective TRPA1 channel antagonist, showed a remarkable reduction in bronchial hyperresponsiveness and a pronounced inhibition of type 2 inflammation. The activation of TRPA 1 induces the release of the neuropeptides CGRP, substance P, and neurokinin A from sensory nerve terminals, and OVA-induced release of neuropeptides is inhibited in TRPA1-deficient mice (76). Therefore, TRPA1 is considered to be associated with antigen-induced neuronal activation and neuropeptide release, which results in the type 2 airway inflammation that characterizes asthma.

A recent study suggested that PNECs are also important sources of neuropeptides, especially CGRP, in the lungs. ILC2s colocalize with PNECs in the lungs, and PNEC-derived CGRP directly promotes ILC2 activation. Moreover, PNECs produce the neurotransmitter GABA, which directly stimulates epithelial cells to induce goblet cell hyperplasia. Accordingly, PNEC-deficient mice exhibit less severe inflammation than control mice in HDMand OVA-induced asthma models (ref. 65 and Figure 3). 
Recent evidence also indicates that enteric cholinergic neuron-derived NMU has a potent activating effect on ILC2s that induces type 2 inflammation in the intestine (60-62). Unlike in the intestine, the population of NMU-expressing neurons in the lungs still remains unclear, and one study showed that NMU is expressed in the neurons of the thoracic dorsal root ganglia, which contain afferent sensory neurons innervating the lungs (61). NMU stimulates lung ILC2s to induce type 2 airway inflammation, and HDM-induced and $N$. brasiliensis-induced lung inflammation was suppressed in NMUR1-deficient mice. These data suggest that neuron-derived NMU also has a role in ILC2-mediated lung inflammation.

Collectively, the conclusions of recent studies indicate that various neurotransmitters and neuropeptides directly affect immune cells, especially Th2 cells and ILC2s, and have a potent effect on type 2 inflammation in the pathophysiology of asthma.

\section{Summary and future directions}

Building on seminal studies conducted decades ago, the study of neuro-immune crosstalk has become an active research area in the field of both immunology and neurobiology. The positivefeedback loop between the neuronal and immune systems in local tissues appears to be an amplification mechanism to induce potent immune reactions. However, excess inflammation might cause health problems, such as allergic and inflammatory diseases. Therefore, the regulation of neuro-immune interaction is a potential therapeutic target for these conditions.

To expand this research area in the future, it could be beneficial to define the activation mechanisms of peripheral neurons in the context of allergy, inflammation, and infection, test the influence of neuropeptides and neurotransmitters on the immune systems, and examine the different roles of neuroimmune crosstalk in various tissues and the context of inflam- mation. Regarding the potential of therapeutically targeting neuro-immune pathways, recent studies have shown that an anti-IL-31 receptor antibody, nemolizumab, successfully improves pruritus in patients with atopic dermatitis (77). Furthermore, ion channels play an essential role in the activation of sensory neurons, and TRP channel inhibitors are also promising candidates for various neuron-related symptoms and diseases (78). Neuropeptides, neurotransmitters, or their receptors are also potential targets for neuro-immune interaction, including VIP, CGRP, substance P, NMU, $\beta 2 A R$, and nAChR. However, we have to interpret the results of rodent-based studies carefully because of species differences in these pathways. For example, substance $\mathrm{P}$ activates mast cells and induces skin inflammation in mice, but the results of clinical studies using substance $\mathrm{P}$ receptor (NK-1R) antagonists were equivocal in human studies (79). Notwithstanding these challenges, future translational efforts to study the effects of local neuro-immune interactions in human health and disease are certainly warranted.

\section{Acknowledgments}

The work was supported by grants from the MSD Life Science Foundation (to HK), the Japan Society for the Promotion of Science (JSPS) Overseas Research Fellowships (to HK), the NIH (AI074878, AI095466, AI095608 and AI102942 to DA), Cure for IBD (to DA), the Burroughs Wellcome Fund (to DA), the Rosanne H. Silbermann Foundation (to DA), and the Crohn's \& Colitis Foundation (to DA).

Address correspondence to: David Artis, Weill Cornell Medicine, Cornell University, Joan and Sanford I. Weill Department of Medicine, Belfer Research Building, Room 724 (box 210), 413 East 69th Street, New York, New York 10021, USA. Phone: 646.962.6291; Email: dartis@med.cornell.edu.
1. Borovikova LV, et al. Vagus nerve stimulation attenuates the systemic inflammatory response to endotoxin. Nature. 2000;405(6785):458-462.

2. Undem BJ, Taylor-Clark T. Mechanisms underlying the neuronal-based symptoms of allergy. J Allergy Clin Immunol. 2014;133(6):1521-1534.

3. Veiga-Fernandes H, Artis D. Neuronal-immune system cross-talk in homeostasis. Science. 2018;359(6383):1465-1466.

4. Chesné J, Cardoso V, Veiga-Fernandes H. Neuro-immune regulation of mucosal physiology. Mucosal Immunol. 2019;12(1):10-20.

5. Moro K, et al. Innate production of $\mathrm{T}(\mathrm{H}) 2$ cytokines by adipose tissue-associated c-Kit(+)Sca-1(+) lymphoid cells. Nature. 2010;463(7280):540-544.

6. Neill DR, et al. Nuocytes represent a new innate effector leukocyte that mediates type-2 immunity. Nature. 2010;464(7293):1367-1370.

7. Price AE, et al. Systemically dispersed innate IL-13-expressing cells in type 2 immunity. Proc Natl Acad Sci U S A. 2010;107(25):11489-11494.

8. Pongratz G, Straub RH. The sympathetic nervous response in inflammation. Arthritis Res Ther. 2014;16(6):504.

9. Scanzano A, Cosentino M. Adrenergic regulation of innate immunity: a review. Front Pharmacol. 2015;6:171.

10. Bosmans G, Shimizu Bassi G, Florens M, Gonzalez-Dominguez E, Matteoli G, Boeckxstaens GE. Cholinergic modulation of type 2 immune responses. Front Immunol. 2017;8:1873.

11. Kong WL, Peng YY, Peng BW. Modulation of neuroinflammation: Role and therapeutic potential of TRPV1 in the neuro-immune axis. Brain Behav Immun. 2017;64:354-366.

12. Kang K, et al. Analysis of Drosophila TRPA1 reveals an ancient origin for human chemical nociception. Nature. 2010;464(7288):597-600.

13. Karashima Y, et al. TRPA1 acts as a cold sensor in vitro and in vivo. Proc Natl Acad Sci US A. 2009;106(4):1273-1278.

14. Bautista DM, et al. The menthol receptor TRPM8 is the principal detector of environmental cold. Nature. 2007;448(7150):204-208.

15. Pinho-Ribeiro FA, Verri WA, Chiu IM. Nociceptor sensory neuron-immune interactions in pain and inflammation. Trends Immunol. 2017;38(1):5-19.

16. Oetjen LK, et al. Sensory neurons co-opt classical immune signaling pathways to mediate chronic itch. Cell. 2017;171(1):217-228.e13.

17. Wilson SR, et al. The epithelial cell-derived atopic dermatitis cytokine TSLP activates neurons to induce itch. Cell. 2013;155(2):285-295.

18. Cevikbas F, et al. A sensory neuron-expressed IL-31 receptor mediates T helper cell-dependent itch: involvement of TRPV1 and TRPA1. J Allergy Clin Immunol. 2014;133(2):448-460.

19. Janelsins BM, et al. Neurokinin-1 receptor agonists bias therapeutic dendritic cells to induce type 1 immunity by licensing host dendritic cells to produce IL-12. Blood. 2013;121(15):2923-2933.

20. O'Connor TM, O'Connell J, O'Brien DI, Goode T, Bredin CP, Shanahan F. The role of substance P in inflammatory disease. JCell Physiol. 2004;201(2):167-180.

21. Lim JE, Chung E, Son Y. A neuropeptide, substance-P, directly induces tissue-repairing M2 like macrophages by activating the PI3K/Akt/ mTOR pathway even in the presence of IFN $\gamma$. Sci Rep. 2017;7(1):9417.

22. Assas BM, Pennock JI, Miyan JA. Calcitonin gene-related peptide is a key neurotransmitter in the neuro-immune axis. Front Neurosci. 2014;8:23.

23. Delgado M, Ganea D. Vasoactive intestinal peptide: a neuropeptide with pleiotropic immune functions. Amino Acids. 2013;45(1):25-39. 
24. Ganea D, Rodriguez R, Delgado M. Vasoactive intestinal peptide and pituitary adenylate cyclase-activating polypeptide: players in innate and adaptive immunity. Cell Mol Biol (Noisyle-grand). 2003;49(2):127-142.

25. Granstein RD, Wagner JA, Stohl LL, Ding W. Calcitonin gene-related peptide: key regulator of cutaneous immunity. Acta Physiol (Oxf). 2015;213(3):586-594.

26. Steinhoff $M$, et al. Agonists of proteinase-activated receptor 2 induce inflammation by a neurogenic mechanism. Nat Med. 2000;6(2):151-158.

27. Forsythe P, Bienenstock J. The mast cell-nerve functional unit: a key component of physiologic and pathophysiologic responses. Chem Immunol Allergy. 2012;98:196-221.

28. Wiesner-Menzel L, Schulz B, Vakilzadeh F, Czarnetzki BM. Electron microscopical evidence for a direct contact between nerve fibres and mast cells. Acta Derm Venereol. 1981;61(6):465-469.

29. Sugiura H, Maeda T, Uehara M. Mast cell invasion of peripheral nerve in skin lesions of atopic dermatitis. Acta Derm Venereol Suppl (Stockh). 1992;176:74-76.

30. Järvikallio A, Harvima IT, Naukkarinen A. Mast cells, nerves and neuropeptides in atopic dermatitis and nummular eczema. Arch Dermatol Res. 2003;295(1):2-7.

31. Oetjen LK, Kim BS. Interactions of the immune and sensory nervous systems in atopy. FEBS J. 2018;285(17):3138-3151.

32. Choi JE, Di Nardo A. Skin neurogenic inflammation. Semin Immunopathol. 2018;40 (3):249-259.

33. Steinhoff M, Buddenkotte J, Lerner EA. Role of mast cells and basophils in pruritus. Immunol Rev. 2018;282(1):248-264.

34. Rosa AC, Fantozzi R. The role of histamine in neurogenic inflammation. Br J Pharmacol. 2013;170(1):38-45.

35. Kakurai M, Monteforte R, Suto H, Tsai M, Nakae $\mathrm{S}$, Galli SJ. Mast cell-derived tumor necrosis factor can promote nerve fiber elongation in the skin during contact hypersensitivity in mice. Am J Pathol. 2006;169(5):1713-1721.

36. Ohmura T, Hayashi T, Satoh Y, Konomi A, Jung B, Satoh H. Involvement of substance P in scratching behaviour in an atopic dermatitis model. Eur JPharmacol. 2004;491(2-3):191-194.

37. Sawatzky DA, et al. Eosinophil adhesion to cholinergic nerves via ICAM-1 and VCAM- 1 and associated eosinophil degranulation. Am J Physiol Lung Cell Mol Physiol. 2002;282(6):L1279-L1288.

38. Jacoby DB, Gleich GJ, Fryer AD. Human eosinophil major basic protein is an endogenous allosteric antagonist at the inhibitory muscarinic M2 receptor. J Clin Invest. 1993;91(4):1314-1318.

39. Dunzendorfer S, Meierhofer C, Wiedermann CJ. Signaling in neuropeptide-induced migration of human eosinophils. JLeukoc Biol. 1998;64(6):828-834.

40. Drake MG, et al. Eosinophil and airway nerve interactions in asthma. J Leukoc Biol. 2018;104(1):61-67.

41. Muller PA, et al. Crosstalk between muscularis macrophages and enteric neurons regulates gastrointestinal motility. Cell. 2014;158(2):300-313.

42. Gabanyi I, Muller PA, Feighery L, Oliveira TY, Costa-Pinto FA, Mucida D. Neuro-immune inter- actions drive tissue programming in intestinal macrophages. Cell. 2016;164(3):378-391.

43. Zeng W, et al. Sympathetic neuro-adipose connections mediate leptin-driven lipolysis. Cell. 2015;163(1):84-94.

44. Wolf Y, et al. Brown-adipose-tissue macrophages control tissue innervation and homeostatic energy expenditure. Nat Immunol. 2017;18(6):665-674.

45. Jung S. Macrophages and monocytes in 2017: Macrophages and monocytes: of tortoises and hares. Nat Rev Immunol. 2018;18(2):85-86.

46. Pirzgalska RM, et al. Sympathetic neuronassociated macrophages contribute to obesity by importing and metabolizing norepinephrine. Nat Med. 2017;23(11):1309-1318.

47. Veres TZ, et al. Spatial interactions between dendritic cells and sensory nerves in allergic airway inflammation. Am J Respir Cell Mol Biol. 2007;37(5):553-561.

48. Riol-Blanco L, et al. Nociceptive sensory neurons drive interleukin-23-mediated psoriasiform skin inflammation. Nature. 2014;510(7503):157-161.

49. Rochlitzer $S$, et al. The neuropeptide calcitonin gene-related peptide affects allergic airway inflammation by modulating dendritic cell function. Clin Exp Allergy. 2011;41(11):1609-1621.

50. Kashem SW, Riedl MS, Yao C, Honda CN, Vulchanova L, Kaplan DH. Nociceptive sensory fibers drive interleukin-23 production from $\mathrm{CD} 301 \mathrm{~b}^{+}$ dermal dendritic cells and drive protective cutaneous immunity. Immunity. 2015;43(3):515-526.

51. Hosoi J, et al. Regulation of Langerhans cell function by nerves containing calcitonin gene-related peptide. Nature. 1993;363(6425):159-163.

52. Ding W, Stohl LL, Wagner JA, Granstein RD. Calcitonin gene-related peptide biases Langerhans cells toward Th2-type immunity. J Immunol. 2008;181(9):6020-6026.

53. Mathers AR, Tckacheva OA, Janelsins BM, Shufesky WJ, Morelli AE, Larregina AT. In vivo signaling through the neurokinin 1 receptor favors transgene expression by Langerhans cells and promotes the generation of Th1- and Tc1-biased immune responses. JImmunol. 2007;178(11):7006-7017.

54. Spits $\mathrm{H}$, et al. Innate lymphoid cells - a proposal for uniform nomenclature. Nat Rev Immunol. 2013;13(2):145-149.

55. Kabata H, Moro K, Koyasu S, Asano K. Group 2 innate lymphoid cells and asthma. Allergol Int. 2015;64(3):227-234.

56. von Moltke J, Ji M, Liang HE, Locksley RM. Tuft-cell-derived IL-25 regulates an intestinal ILC2-epithelial response circuit. Nature. 2016;529(7585):221-225.

57. Gerbe F, et al. Intestinal epithelial tuft cells initiate type 2 mucosal immunity to helminth parasites. Nature. 2016;529(7585):226-230.

58. Howitt MR, et al. Tuft cells, taste-chemosensory cells, orchestrate parasite type 2 immunity in the gut. Science. 2016;351(6279):1329-1333.

59. Kohanski MA, et al. Solitary chemosensory cells are a primary epithelial source of IL-25 in patients with chronic rhinosinusitis with nasal polyps. J Allergy Clin Immunol. 2018;142(2):460-469.e7.

60. Klose CSN, et al. The neuropeptide neuromedin U stimulates innate lymphoid cells and type 2 inflammation. Nature. 2017;549(7671):282-286.
61. Wallrapp A, et al. The neuropeptide NMU amplifies ILC2-driven allergic lung inflammation. Nature. 2017;549(7672):351-356.

62. Cardoso V, et al. Neuronal regulation of type 2 innate lymphoid cells via neuromedin U. Nature. 2017;549(7671):277-281.

63. Galle-Treger L, et al. Nicotinic acetylcholine receptor agonist attenuates ILC2-dependent airway hyperreactivity. Nat Commun. 2016;7:13202.

64. Moriyama S, et al. $\beta 2$-adrenergic receptormediated negative regulation of group 2 innate lymphoid cell responses. Science. 2018;359(6379):1056-1061.

65. Sui P, et al. Pulmonary neuroendocrine cells amplify allergic asthma responses. Science. 2018;360(6393):eaan8546.

66. Nussbaum JC, et al. Type 2 innate lymphoid cells control eosinophil homeostasis. Nature. 2013;502(7470):245-248.

67. Ibiza S, et al. Glial-cell-derived neuroregulators control type 3 innate lymphoid cells and gut defence. Nature. 2016;535(7612):440-443.

68. Brightling CE, Bradding P, Symon FA, Holgate ST, Wardlaw AJ, Pavord ID. Mast-cell infiltration of airway smooth muscle in asthma. N Engl JMed. 2002;346(22):1699-1705.

69. Chachi L, et al. Increased $\beta 2$-adrenoceptor phosphorylation in airway smooth muscle in severe asthma: possible role of mast cell-derived growth factors. Clin Exp Immunol. 2018;194(2):253-258.

70. Moulton BC, Fryer AD. Muscarinic receptor antagonists, from folklore to pharmacology; finding drugs that actually work in asthma and COPD. Br J Pharmacol. 2011;163(1):44-52.

71. Su X, Matthay MA, Malik AB. Requisite role of the cholinergic alpha7 nicotinic acetylcholine receptor pathway in suppressing Gram-negative sepsis-induced acute lung inflammatory injury. JImmunol. 2010;184(1):401-410.

72. Tränkner D, Hahne N, Sugino K, Hoon MA, Zuker C. Population of sensory neurons essential for asthmatic hyperreactivity of inflamed airways. Proc Natl Acad Sci US A. 2014;111(31):11515-11520.

73. Talbot S, et al. Silencing nociceptor neurons reduces allergic airway inflammation. Neuron. 2015;87(2):341-354.

74. Tomaki M, et al. Elevated substance P content in induced sputum from patients with asthma and patients with chronic bronchitis. Am J Respir Crit Care Med. 1995;151(3 pt 1):613-617.

75. Kay AB, et al. Airway expression of calcitonin gene-related peptide in T-cell peptide-induced late asthmatic reactions in atopics. Allergy. 2007;62(5):495-503.

76. Caceres AI, et al. A sensory neuronal ion channe essential for airway inflammation and hyperreactivity in asthma. Proc Natl Acad Sci US A. 2009;106(22):9099-9104.

77. Ruzicka T, et al. Anti-interleukin-31 receptor A antibody for atopic dermatitis. $N$ Engl J Med. 2017;376(9):826-835

78. Moran MM. TRP channels as potential drug targets. Annu Rev Pharmacol Toxicol. 2018;58:309-330.

79. Voisin T, Bouvier A, Chiu IM. Neuro-immune interactions in allergic diseases: novel targets for therapeutics. Int Immunol. 2017;29(6):247-261. 